\title{
Kräuterbücher (8): Eine neue Sicht der Wissenschaft - wie das Mikroskop den Blick der Medizin veränderte
}

\author{
Martin Kluge
}

Nachdem Galileo Galilei (1564-1642) um das Jahr 1609 eines der ersten Mikroskope gebaut hatte und das Mikroskop zum zentralen Instrument wissenschaftlicher Erkenntnisse des 17. und 18. Jahrhunderts aufstieg, war der englische Universalgelehrte Robert Hooke (1635-1703) einer der Ersten, die Bildtafeln zur neuen, sich unter dem Mikroskop auftuenden Welt publizierten. Im Jahr 1665 erschien sein damals weitbekanntes Werk «Micrographia: or, Some physiological descriptions of minute bodies made by magnifying glasses» mit zahlreichen Kupfertafeln zur Mikrowelt der Samen und Früchte, Tiere, Mineralien, Gewebe und Kristalle.

Unter den Buch-Raritäten des ETH-Professors Carl Hartwich (1851-1917), die Gegenstand dieser Artikelreihe sind, befindet sich ein kleiner, ebenfalls mit Kupfertafeln versehener Band aus den Anfängen des Mikroskopierens, der direkt auf die «Micrographia» von Hooke Bezug nimmt: die «Micrographia Nova: Oder Neu-Curieuse Beschreibung Verschiedener kleiner Cörper, Welche Vermittelst eines absonderlichen von dem Authore neuerfundenen Vergrösser-Glases Verwunderlich groß vorgestellet werden» [1]. Verfasst wurde das Werk 1687 von Johann Franz Griendel von Ach (1631-1687). Dieser trat mit 24 Jahren auf elterlichen Wunsch in den Kapuzinerorden ein und lebte als Kapuzinermönch in Salzburg, München, Kitzingen und Würzburg. 1670 verliess er den Orden und eröffnete wenig später in Nürnberg eine Werkstatt, in der er eine Vielzahl optischer Instrumente anbot. 1677 zog er als kurfürstlicher Ingenieur nach Dresden. 1684 siedelte er nach Wien um. Hier entwarf Griendel von Ach ein verbessertes Mikroskop mit drei feststehenden Linsenpaaren (Abb. 1). Dieses veröffentlichte er zusammen mit 52 mikroskopischen Beobachtungen auf entsprechend vielen Kupfertafeln in besagtem Titel. Analog zu seinem Vorbild, der «Micrographia» von Hooke, mikroskopierte er Nadelspitzen, Gewebe, eine Ameise, Fliegenköpfe und diverse Läuse und Würmer. Beispielsweise beschreibt er einen Wurm, der «in einer Erbsen ge-
Abb. 1. Darstellung des Mikroskops von Johann Franz Griendel von Ach in seiner "Micrographia Nova», 1687 (Foto: Pharmazie-Historisches Museum Basel).

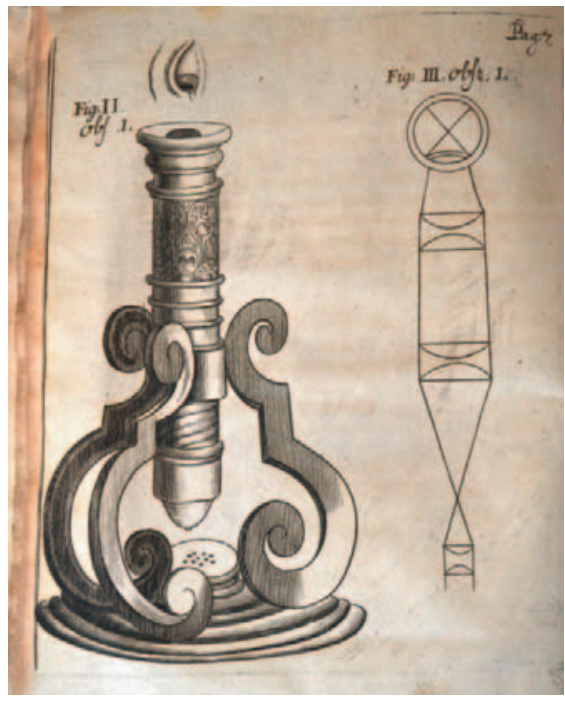

wachsen» mit den Worten: «Das Vergrösser-Glaß stellet hier vor Augen / den abscheulichsten Wurm/ nemlich einen so in Erbsen gewachsen ist / er ist ein Wunder-Thier drey Schuh $(=95 \mathrm{~cm})$ lang/ und sechs Zoll $(=16 \mathrm{~cm})$ breit/ und in dem Vergrösser-Glas/ erschrecklicher anzusehen/ als ein Pantherthier aus India/» [2]. Auch die Ameise «weißet sich durch das Vergrößer-Glas/ ein WunderThier zu seyn; massen dieselbe erscheinet/ wie ein grausamer Ochs» [3].

Solche Formulierungen lassen erahnen, dass Griendel von Ach als Ingenieur im Umfeld des Wiener Hofs weniger an einer exakten Naturbeschreibung interessiert zu sein schien als an höfischer Übertreibung und barocker Wunderkammer-Ästhetik (Abb. 2). Die unter dem Mikroskop beobachtete Begegnung einer Laus mit einem Floh verglich er gar mit einer Tierhatz im römischen Amphitheater: Bei der Begegnung habe sich «zwischen diesen zwei geharnischten und gestachelten Unthieren/ ein dermassen hefftiger Streit erhoben/ daß man es mit Verwunderung nicht genugsam beschreiben kann/ wie sie ihre stachlichte Füsse und Klauen einander in den Laib ge-

\section{KARGER}

Fax +497614520714

Information@Karger.com

www.karger.com
() 2015 S. Karger GmbH, Freiburg

$1015-0684 / 15 / 0273-0159 \$ 39.50 / 0$
Martin Kluge

Pharmazie-Historisches Museum der Universität Basel

Totengässlein 3, 4051 Basel, Schweiz

martin.kluge@unibas.ch

www.pharmaziemuseum.ch 
Abb. 2. Darstellung eines Käswurms in hartem Käse aus der «Micrographia Nova» (Foto: PharmazieHistorisches Museum Basel).

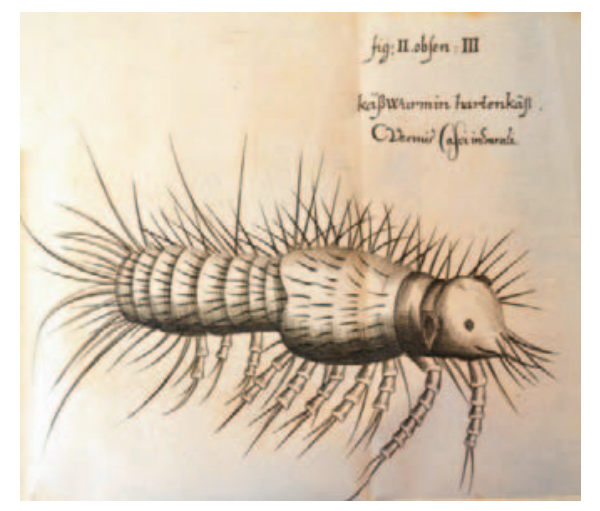

setzt/... als ob anstatt der Laus ein Crocodill/ und deß Flohes/ wie ein geharnischtes Rinoceros mit einander kämpfften/ und streitteten. Diese kleinen Unthier und Unziefer / so aus dem Schweiß des Menschen hervor wachsen / wann sie so groß wären als sie im Vergrösser-Glaß erscheinen / würden gewißlich grössere Lust und Kurtzweil machen/ als etwan der Löwen und Bären-Streit» [4].

Die von ihm erhoffte wissenschaftliche Anerkennung für seine Leistung blieb allerdings aus, und aufgrund anhaltender Probleme beim Zentrieren der zahlreichen Linsen setzte sich auch das Prinzip seines Mikroskops in der Praxis nicht durch. Zusammengesetze Mikroskope des ausgehenden 17. Jahrhunderts ermöglichten kaum mehr als eine 100-fache Vergrösserung. Die erzeugten Bilder waren zudem oft von Lufteinschlüssen im Glas getrübt und infolge der handgeschliffenen Linsen mehr oder weniger verzerrt.

Erfolgreicher als Griendel von Ach in Wien wirkte Antoni van Leeuwenhoek (1632-1723) [5-7] als Linsenschleifer und Instrumentenbauer in Delft. Der Sohn eines Korbmachers stieg nach einer Lehre als Buchhalter erfolgreich in den Tuchhandel ein und sicherte sich so ein Leben in gewissem Wohlstand. Finanziell abgesichert konnte van Leeuwenhoek es sich bald leisten, Zeit in die Entwicklung verbesserter Mikroskope zu investieren. Als Linsenschleifer und Instrumentenbauer war van Leeuwenhoek Autodidakt, fertigte aber mit einer von ihm erfundenen und geheim gehaltenen Methode winzige Glaskugeln, die er zu Linsen schliff. Diese montierte er zwischen zwei Messingplatten und befestigte die zu mikroskopierenden Objekte an einer verstellbaren Nadelspitze. Da kugelförmige Linsen stärker vergrössern und ein klareres Bild ergeben als linsenförmige, dafür aber geringere Brennweiten aufweisen, gelangen ihm mit seinen winzigen $\mathrm{Ku}$ gellinsen Vergrösserungen um das 270-Fache. Allerdings mussten die Mikroskope aufgrund ihrer Brennweite von rund einem Millimeter dicht an das Auge gehalten werden. Diese einfachen Mikroskope übertrafen die Leistung selbst mehrlinsiger Mikroskope bei Weitem. Über 500 derartige Mikroskope soll van Leeuwenhoek im Laufe seines Lebens gefertigt haben.

\section{Revolutionäre Erkenntnisse}

Offensichtlich von Robert Hookes «Micrographia» inspiriert, begann van Leeuwenhoek mit eigenen mikroskopischen Beobachtungen. Unter anderem konnte er im Jahr 1668 mit seinem Mikroskop die Entdeckung des Kapillarsystems im Blutkreislauf bestätigen, den William Harvey (1578-1657) im Jahr 1628 bereits postuliert hatte. Dieser widersprach damit der bis anhin gültigen Lehrmeinung, dass das Blut laufend in der Leber produziert und durch Kontraktion der Arterien in Bewegung versetzt würde. Harvey fehlten allerdings damals die technischen Möglichkeiten, seine These zu belegen. Erst 1661 gelang es dem italienische Anatom Marcello Malpighi, unter dem Mikroskop Kapillare nachzuweisen und somit den ausstehenden Beweis für die Richtigkeit der Theorie Harveys zu erbringen. Dank seines verbesserten Mikroskops beobachtete van Leeuwenhoek sogar das Zirkulieren roter Blutkörperchen durch die Kapillaren eines Kaninchenohrs und eines Froschbeins. Doch statt der erhofften Anerkennung zog er als Autodidakt ohne akademische Ausbildung lediglich den Spott der wissenschaftlichen Welt auf sich. In dieser Situation wurde Reinier de Graaf, ein ebenfalls aus Delft stammendes Mitglied der Londoner Royal Society, auf van Leeuwenhoek aufmerksam und führte ihn 1673 mit seinen Mikroskopen in wissenschaftliche Kreise ein, die seine Beobachtungen verifizierten. Seither lösten seine Entdeckungen kontroverse Diskussionen aus und revolutionierten das medizinische Verständnis: 1674 lieferte van Leeuwenhoek die erste genaue Beschreibung der roten Blutkörperchen, die zuvor von Jan Swammerdam unter dem Mikroskop eher schemenhaft entdeckt wurden. Des Weiteren beschrieb er die Querstreifung der Muskulatur und das Netzwerk der Zellen am Herzmuskel.

Im September des gleichen Jahres mikroskopierte er eine Wasserprobe eines nahegelegenen Moorsees und beschrieb in einem Brief [8], im Wasser seien ...

«(...) sehr viele kleine Animalcules, wovon einige rundlich, andere hingegen ein wenig grösser, in der Form eines Ovals waren. Auf diesen sah ich zwei kleine Beine neben dem Ohr, und zwei kleine Flossen am hintersten Ende des Körpers. Andere, gering an der Zahl, waren etwas länger als ein Oval, und bewegten sich langsam. Diese Animalcules hatten verschiedene Farben, einige waren weisslich und transparent; andere mit grünen und sehr glänzenden kleinen Schuppen. Andere waren in der Mitte grün, vorne und hinten weiss, andere wiederum aschgrau. Und die Bewegungen der meisten dieser Tierchen im Wasser war so schnell, und verschiedenartig, auf- und abwärts und rundherum, dass es wunderlich anzusehen war: Und ich schätze dass einige dieser kleinen Kreaturen ungefähr tausendmal kleiner waren als das kleinste das ich je zuvor gesehen habe, sei es auf der Käserinde, in Weizenmehl, im Schimmel oder ähnlichem.»

Diese von ihm beobachteten Protozoen (Einzeller) wies er im Teichwasser, im Regenwasser und im menschlichen Speichel nach. 1683 entdeckte er Bakterien im Zahnbelag. 
Abb. 3. «Generierung eines Froschs», dargestellt in der «Micrographia Nova» von Johann Franz Griendel von Ach (Foto: Pharmazie-Historisches Museum Basel).

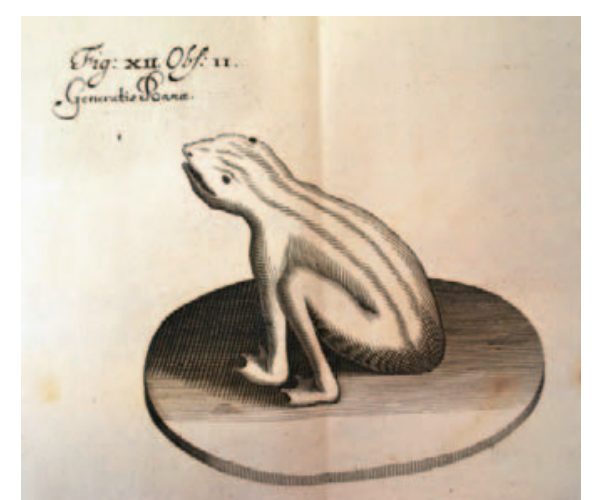

Van Leeuwenhoeks animalcules (Tierchen) ebneten nicht nur der modernen Immunologie den Weg. Auch Vertreter der mittelalterlichen «Spontanzeugung», die sich vehement gegen den von William Harvey postulierten Grundsatz «Omne vivum ex ovo» («Jedes Leben entsteht aus einem Ei») wandten, erlebten neuen Aufwind. Für sie war es selbstverständlich, dass einfache Lebewesen wie Würmer oder Insekten direkt aus Dreck entstünden, wie es das «spontane» Auftreten von Maden auf faulem Fleisch nahelege. Selbst wenn nun die Erzeugung von Würmern und Fliegen aus Eiern mikroskopisch belegt war, so schien es dennoch unvorstellbar, dass so primitive Wesen wie van Leeuwenhoeks «Tierchen» in der Lage seien, Eier zu legen.

Auch Johann Franz Griendel von Ach glaubte an diese Spontanzeugung und beschrieb kurzerhand in seiner «Micrographia Nova», wie er die spontane Entstehung eines Froschs aus Wasser unter seinem Mikroskop beobachtet habe (Abb. 3) [9]:

«Generirung eines Frosches: Ich habe zuletzt nicht weniger eines Frosches wunderliche Hervorbringung an das Welt-Licht vorstellen wollen/ welche ich durch das Vergrösser-Glaß observieret. Einsmals nahme ich einen Tropfen Mayen-Thau/ und legte ihn unter das Vergrösser-Glaß / da nam ich in acht wie er sich anfienge zu fermentiren; den andern Tag sahe ich weiter darnach/ und fande schon ein Corpus mit einem ungestallten Kopff/ setzte es beyseits/ und als ich den dritten Tag wiederum selbiges besahe/ konnte ich schon abmercken/ daß es die Gestalt mit einem grossen Kopff/ und Füssen wie ein Laub-Frosch angenommen hatte. Die Fig. XII. stellt alles deutlich vor Augen».

\section{Mikroben und Bazillen}

Von der Entdeckung der animalcules bis zur Erkenntnis, in ihnen auch mögliche Krankheitsursachen zu sehen, war noch ein weiter Weg. Bis sich die «Keimtheorie» von Louis Pasteur (1822-1895) durchsetzen konnte, wonach Mikroorganismen Krankheiten verursachen und über Tröpfchen, verunreinigtes Wasser oder kontaminierte Nahrungsmittel übertragen werden können, dauerte es noch bis in das ausgehende 19. Jahrhundert. 1876 entdeckte Robert Koch
Abb. 4. Darstellung der Kamille (Ausschnitt) [21] (Foto: Pharmazie-Historisches Museum Basel).

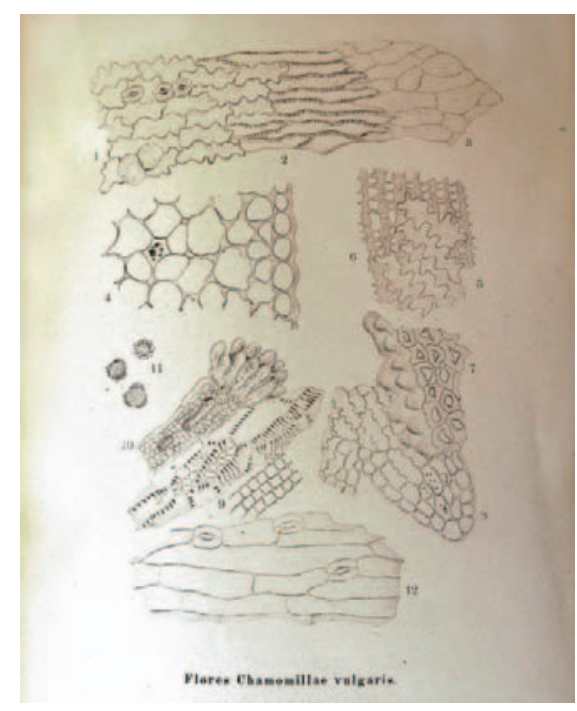

(1843-1910) den Milzbranderreger und beschrieb dessen Lebenszyklus, 1882 denjenigen der Tuberkulose. 1884 identifizierte Friedrich Loeffler (1852-1915) den Erreger der Diphtherie, und 1894 isolierte Alexandre Émile Jean Yersin (1863-1943) den Pesterreger, wodurch er erstmals die Infektionskette der Pestepidemie durch den Biss des Rattenflohs und deren Übertragung vom Tier auf den Menschen lückenlos nachweisen konnte.

\section{Die Geburt der Pharmakognosie}

Das Mikroskop gehörte nun zum unumgänglichen Arbeitsinstrument medizinischer Forschung und wurde auch zur exakten Bestimmung der Heilpflanzen und ihrer Teile in der pharmazeutischen Botanik unentbehrlich. Es war die Geburtsstunde des Fachs Pharmakognosie (von griechisch Pharmakon (Heilmittel); gignoskein (erkennen)). Die «Drogenkunde» umfasste die Suche nach Wirkstoffen in lebendem Material (also in Tieren und Pflanzen) sowie die Analyse der daraus gewonnenen Substanzen. Unzählige Handbücher zur mikroskopischen Bestimmung erschienen als Folge auf dem Markt (Abb. 4). $\mathrm{Zu}$ den bedeutenden Arbeiten in diesem Fach zählen unter anderem die Werke von Friedrich August Flückiger (1828-1894) und Alexander Tschirch (1856-1939) in Bern. Auch Carl Hartwich als Professor für Pharmakognosie am damaligen Eidgenössischen Polytechnikum in Zürich, der heutigen ETH, gab entsprechende Handbücher heraus.

Das Augenmerk des neuen Fachs richtete sich nun nicht mehr auf die Kenntnis und Anwendung der Heilpflanzen an sich, sondern verlagerte sich auf die Suche und Analyse der darin enthaltenen Wirkstoffe.

Die Idee des Wirkstoffs geht zurück bis in das 16. Jahrhundert. Paracelsus (1493-1541) [10] schrieb den natür- 


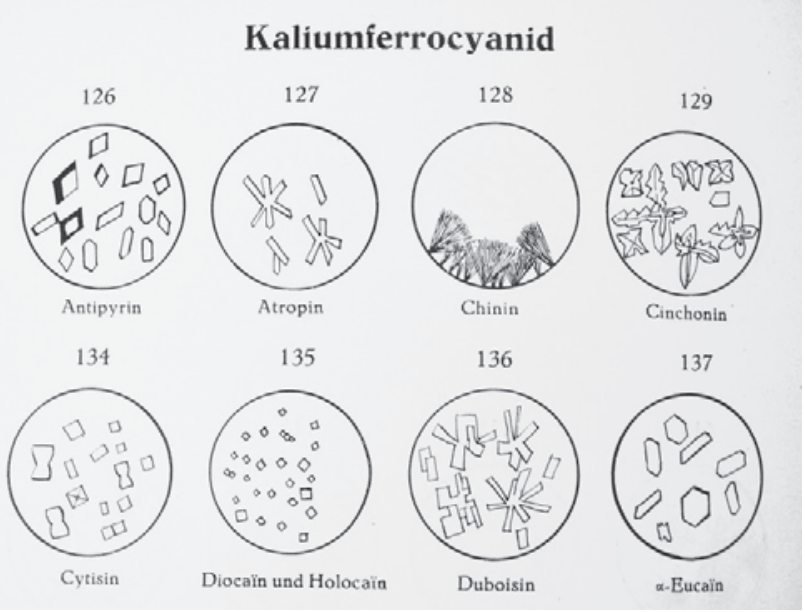

Abb. 5. Vergleichstabelle zu Kristallisationsformen von Alkaloiden (Ausschnitt) [22] (Foto: Pharmazie-Historisches Museum Basel).

lichen Dingen wie Pflanzen und Mineralien heilende Tugenden und Kräfte zu. Diese seien in Form eines Arcanum in den Dingen verborgen und könnten als Heilmittel von dem Übrigen getrennt werden $[11,12]$. Damit legte er den Grundstein für die Idee eines in der Pflanze verborgenen Wirkstoffs. Die Herausforderung der Pharmazie der nachfolgenden Jahrhunderte bestand im Wesentlichen darin, aus den tierischen und pflanzlichen Drogen, in denen unzählige Stoffe enthalten sein können, einzelne dieser Stoffe zu extrahieren, zu analysieren und diese auf ihre pharmakologische Wirkung hin zu untersuchen. Exemplarisch sei diese Problematik anhand der Diskussion um den Wirkstoff im Opium dargestellt, zu dem unter anderem auch Carl Hartwich forschte $[13,14]$ und deren Geschichte gut in seiner Bibliothek dokumentiert ist:

Grosse Anstrengungen, den Wirkstoff des Opiums zu ergründen, wurden bereits im 18. Jahrhundert unternommen [15]:

«Es ist eine große Mannigfaltigkeit von Versuchen ersonnen und gemacht worden, um die medizinischen Kräfte und Eigenschaften des Mohnsafts zu entdecken. Einige haben solches durch eine chemische Zergliederung untersuchet, andere haben es entweder mit frisch gelassenem Blut vermischt, oder haben das laudanum liquidum (= Opium) in die Blutgefäße lebendiger Thiere eingespritzet; eine dritte Art von Schriftstellern haben aus seinem Geschmack und Geruch allerhand Muthmaßungen von dessen Eigenschaft gemacht».

An anderem Ort heisst es: «Von seinem würkendem Wesen sagt einer, es wäre ein Oleum; ein ander, es wäre ein Sal volantile; ein dritter, es wäre ein Sal acidum, der vierdte, es wäre ein Mercurius vegetabilis, wieder andere es wäre ein Sulphur, ...» [16]. Die narkotische Wirkung wurde dabei oftmals Schwefel zugeschrieben, der sich aber im Opium nicht nachweisen liess. Folgerichtig konstruierten einige Autoren eine Art flüchtigen Schwefel als Narkotikum, ein sulfur immaturum bzw. sulfur volatile [17].

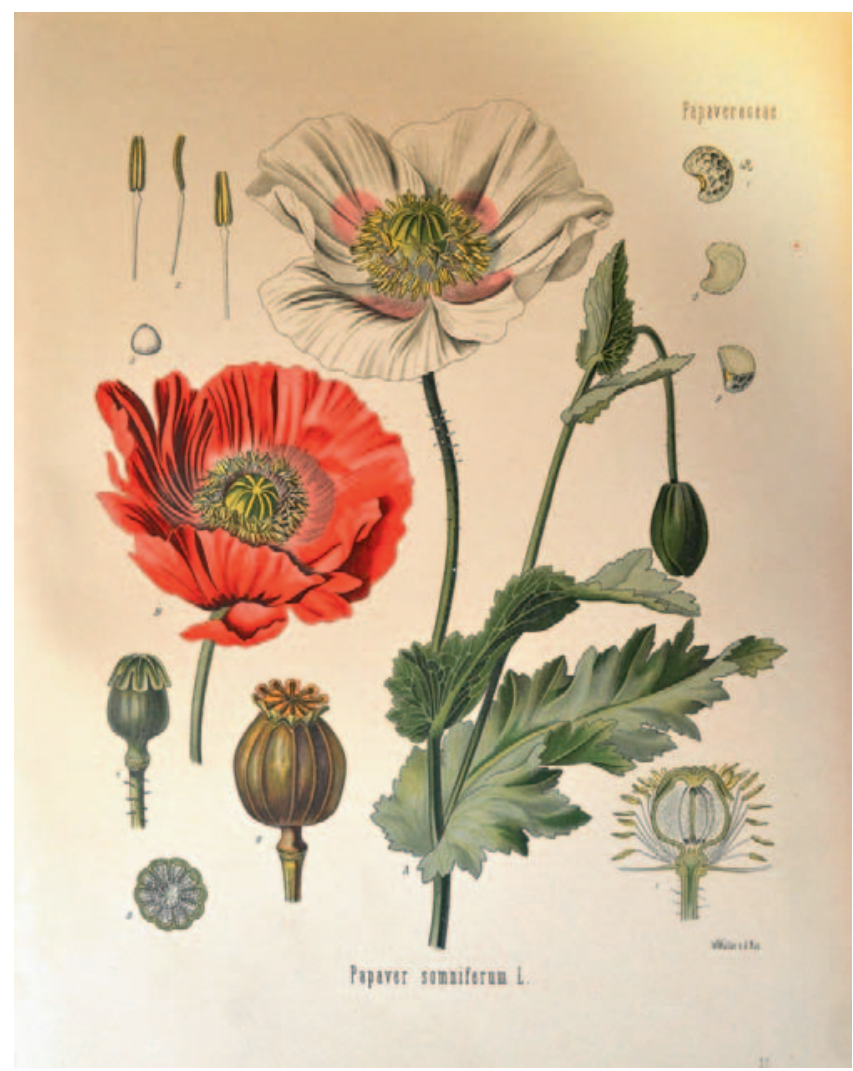

Abb. 6. Darstellung des Mohns [20] (Foto: Pharmazie-Historisches Museum Basel).

Ähnlich probierte auch der Pariser Apotheker JeanFrancois Derosne im Jahr 1803 die Extraktion eines Opiumöls mit Alkohol und Wasser, das er anschliessend zu Opiumsalz zu kristallisieren versuchte [18]. Da Rohopium in der Regel lediglich 12\% Morphin enthält, kam Derosne bei seinen Kristallisationsversuchen zu widersprüchlichen Ergebnissen. Friedrich Wilhelm Sertürner (1783-1841), Apotheker in Paderborn, postulierte 1805 hingegen eine Art Opiumsäure. Demnach bestünde Opium aus einer basischen Substanz, ähnlich dem Ammoniak und den Alkaliverbindungen. Mittels verschiedener Säuren gelang es ihm, Salze aus dem Opium zu kristallisieren, die er nach dem Gott des Traumes Morpheus Morphin nannte. Die Ergebnisse publizierte er «mit vorzüglicher Hinsicht auf einen darin neu entdeckten Stoff» [19]. Zur Charakterisierung der von ihm isolierten Morphinsalze bediente er sich des Mikroskops: Er beschrieb ihre Kristallstruktur und verglich sie mit der Struktur der von Derosne 1803 hergestellten Opiumsalze. Später, d.h. 1819, prägte Wilhelm Meissner (1792-1853) für diese neue Stoffgruppe den Begriff «Alkaloide», eine Wortschöpfung aus Al-Kali - nach dem arabischen Wort al-qalya für «Pflanzenasche» und der griechischen Wortendung -oides für «ähnlich».

Die Entdeckung des Morphins als ersten rein hergestellten Wirkstoff und Sertürners Weg, Alkaloide anhand 
ihrer Kristallformen unter dem Mikroskop zu identifizieren (Abb. 5), legten den Grundstein für die Herstellung standardisierter Produkte der pharmazeutischen Industrie und die im 19. Jahrhundert einsetzende Therapie mit Alkaloiden.

Das beginnende Industriezeitalter veränderte auch den Blick auf die Heilpflanzen. Arzneipflanzen wurden nun zur Rohstoffquelle von Wirkstoffen, die man extrahieren, rein darstellen und letztlich als Extrakte auch industriell nutzen konnte. Aus den Kräuterbüchern des 16. Jahrhunderts, die auf die Anwendungen ganzer Pflanzen und Pflanzenteile ausgerichtet waren, wurden im 19. Jahrhundert auf Inhaltsstoffe fokussierte Arzneipflanzenwerke als Grundlage zur Herstellung industrieller Pharmaka. Ein typisches Werk dieser Art ist «Köhler's Medizinalpflanzen» [20], 1887 in Gera gedruckt (Abb. 6). Basierend auf der Arbeit von Hermann Adolph Köhler (1834-1879), der im Februar 1879 einer schweren Erkrankung erlag, gab Gustav Pabst das weiter ausgearbeitete und mit Farblithografien versehene Werk von 1883 an in Einzelfaszikeln heraus. Die erste vollständige Ausgabe erschien 1887. Rund 400 Arzneipflanzen werden in diesem Werk erläutert und mit chromolithographischen Tafeln illustriert, welche die Pflanzen in allen relevanten Teilen, wie Samen, Blüten, Blättern und Früchten, in Aufsichten und Querschnitten abbildeten. Es ist damit eines der bekanntesten Handbücher zu Heilpflanzen des ausgehenden 19. Jahrhunderts. Der genauen botanischen Beschreibung der Pflanze folgen darin die Aufzählung aller offizinell genutzten Pflanzenteile sowie ihrer Inhaltsstoffe und deren chemischen Formeln. Schliesslich folgen industrielle Anwendungsmöglichkeiten und eine Liste bereits im Handel erhältlicher Präparate.

\section{Literatur}

1 de Martin H: Griendel von Ach. Ein Mikroskopiker der Barockzeit. Wien, 1970.

2 Griendel von Ach JF: Micrographia Nova, p 32.

3 Griendel von Ach JF: Micrographia Nova, p 11.

4 Griendel von Ach JF: Micrographia Nova, p 16.

5 Dobell C: Antony van Leeuwenhoek and his 'little Animals'. Amsterdam, 1932.

6 Hintzsche E: Die Anfange der mikroskopischen Anatomi. CIBA-Zeitschrift 9, Februar 1948, pp 4055-4060

7 Hintzsche E: Mikroskope aus dem 17. und 18. Jahrhundert. CIBA-Zeitschrift 10, 1948, pp 4242-4249.

8 Aus der englischen Übersetzung des holländischen Briefs übersetzt, nach Dobell C: Antony van Leeuwenhoek and his little Animals'. Amsterdam, 1932, p 110.

9 Griendel von Ach JF: Micrographia Nova, p 28.

10 Kluge M: Kräuterbücher (5): Einblicke in das Alchemielabor: Zwei bisher unbekannte Alchemistenhandschriften. Schweiz Z Ganzheitsmed 2014;26:350-353.

11 Paracelsus: Archidoxis. 1525.
12 Paracelsus: Das Buch Paragranum. 1529.

13 Hartwich C, Rudio F: Das Opium als Genussmittel. Neujahrsblatt herausgegeben von der Naturforschenden Gesellschaft auf das Jahr 1898, Zürich 1898.

14 Brauckmann B: Kräuterbücher (1): Sammelleidenschaft und Forschungseifer: Zwei Gelehrte aus verschiedenen Jahrhunderten auf den Spuren von Opium und Kaffee. Schweiz Z Ganzheitsmed 2014;26:105-107.

15 Young GJ: Abhandlung vom Opio oder Mohnsafte, auf praktische Bemerkungen gegründet, durch George Young aus dem Engl. übersetzt. Bayreuth, Andreas Lübecks, 1760, pp 5-6.

16 Neumann C: Lectiones publicae von 4 subjectis pharmaceuticis, nehmlich vom succino, opio caryophyllis aromaticis und castoreo. Berlin, 1730, p 100.

17 Neumann C: Lectiones publicae von 4 subjectis pharmaceuticis, nehmlich vom succino, opio caryophyllis aromaticis und castoreo. Berlin, 1730, pp 109-110.
18 Kessler M, et al: Strömung, Kraft und Nebenwirkung. Eine Geschichte der Basler Pharmazie. Neujahrsblatt der Gesellschaft für das Gute und Gemeinnützige, Basel GGG. Basel, 2002.

19 Sertürner FW: Darstellung der reinen Mohnsäure (Opiumsäure) nebst einer Untersuchung des Opiums mit vorzüglicher Hinsicht auf einen darin neu entdeckten Stoff und die dahin gehörigen Bemerkungen. Vom Herrn Sertürner in Paderborn. Journal der Pharmacie 1806;14:33-37.

20 Pabst G: Köhler's Medizinal-Pflanzen in naturgetreuen Abbildungen mit kurz erläuterndem Texte, in 2 Bänden, der dritte Band in Einzelfaszikel. Gera, 1887.

21 Moeller J: Pharmakognostischer Atlas. Mikroskopische Darstellung und Beschreibung der in Pulverform gebräuchlichen Drogen. Berlin, 1892.

22 Amelink F: Schema zur mikrochemischen Identifikation von Alkaloiden. Amsterdam, 1934. 